\title{
Embodied Meter: Hierarchical Eigenmodes in Music-Induced Movement
}

\author{
Petri Toiviainen, Geoff Luck, \\ \& MARC R. ThOMPSON \\ Department of Music, University of Jyväskylä, \\ Jyväskylä, Finland
}

LISTENING TO MUSIC OFTEN IS ASSOCIATED WITH spontaneous body movements frequently synchronized with its periodic structure. The notion of embodied cognition assumes that intelligent behavior does not emerge from mere passive perception, but requires goal-directed interactions between the organism and its environment. According to this view, one could postulate that we may use our bodily movements to help parse the metric structure of music. The aim of this study was to investigate how pulsations on different metrical levels manifest in musicinduced movement. Musicians were presented with a piece of instrumental music in 4/4 time, played at four different tempi ranging from 92 to $138 \mathrm{bpm}$. Participants were instructed to move to the music, and their movements were recorded with a high quality optical motion capture system. Subsequently, signal processing methods and principal components analysis were applied to extract movement primitives synchronized with different metrical levels. We found differences between metric levels in terms of the prevalence of synchronized eigenmovements. For instance, mediolateral movements of arms were found to be frequently synchronized with the tactus level pulse, while rotation and lateral flexion of the upper torso were commonly found to exhibit periods of two and four beats, respectively. The results imply that periodicities on several metric levels are simultaneously present in music-induced movement. This could suggest that the metric structure of music is encoded in these movements.

Received October 30, 2009, accepted April 7, 2010.

Key words: music, movement, synchronization, meter, embodiment

$\mathrm{T}$ HERE IS A STRONG CONNECTION BETWEEN MUSiC and bodily movement. When we listen to a piece of music, we often tap our foot, nod our head, or move our body along with it. In most cultures, music and dance have evolved together (Arom, 1991; Cross, 2003; Wallin, Merker, \& Brown, 2000). Most people report that they move with music (Lesaffre et al., 2008). When listening to music, people tend to walk faster (i.e., take longer strides) than with metronomic stimuli (Styns, van Noorden, Moelants, \& Leman, 2007).

The notion of embodied cognition (Leman, 2008) assumes that intelligent behavior does not emerge from mere passive perception, but requires goal-directed interactions between an organism and its environment. In the field of linguistics, the motor theory of speech perception (Liberman \& Mattingly, 1985) draws from this notion, assuming that speech perception is based on the cognitive system's ability to compute the articulatory gestures that could have produced the received acoustical signal. The notion of embodied music cognition has its roots in the early 20th century and has recently been elaborated by Leman (2008). Leman emphasizes the role of goaldirected actions in music perception. He sees synchronization to music as a form of corporeal imitation, and postulates: "Spontaneous movements [to music]—may be closely related to predictions of local bursts of energy in the musical audio stream, in particular to the beat and the rhythm patterns" (Leman, 2008).

There is evidence that movement is more strongly connected to the auditory system than to the visual system. For instance, tapping to a visual rhythm is more efficiently disrupted with an auditory distractor than vice versa (Repp \& Penel, 2004). Moreover, Patel, Iversen, Chen, and Repp (2005) found that in a tapping task participants failed to synchronize to metrical nonisochronous visual stimuli, while they had no difficulty in synchronizing to similar auditory stimuli.

Music often contains recurring temporal patterns that give rise to a percept of beat. The beat refers to the subjective sense of periodicity in music evoked by temporal regularities in the acoustical signal. The ability to synchronize with musical beat has to date been mostly investigated with finger tapping studies. According to these studies, synchronization ability is spontaneous and accurate (Drake, Penel, \& Bigand, 2000; Large, Fink, \& Kelso, 2002; Snyder \& Krumhansl, 2001; Toiviainen \&

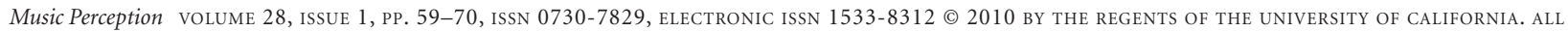
RIGHTS RESERVED. PLEASE DIRECT ALL REQUESTS FOR PERMISSION TO PHOTOCOPY OR REPRODUCE ARTICLE CONTENT THROUGH THE UNIVERSITY OF CALIFORNIA PRESS'S RIGHTS AND PERMISSIONS WEBSITE, HTTP://WWW.UCPRESSJOURNALS.COM/REPRINTINFO.ASP. DOI:10.1525/MP.2010.28.1.59
} 
Snyder, 2003) within a relatively wide range of periods (300-900 ms), and is associated with a preferred pulse period near 500 ms (Fraisse, 1982; Parncutt, 1994; van Noorden \& Moelants, 1999).

When listening to music, we frequently perceive more than one beat level. These beats are often hierarchically organized, with their periods having integer ratios (Palmer \& Krumhansl, 1990). The interaction between these different pulse sensations results in a percept of periodically alternating strong and weak beats, corresponding to the generally accepted definition of meter (Lerdahl \& Jackendoff, 1983). Metrical structure is normally derived from the accent structure of music, which again emerges from various sources such as the duration, loudness, and pitch of tones, and harmonic changes. Most music has either a duple (every second beat accented) or a triple meter (every third beat accented).

While music certainly induces movement, there also is some evidence to suggest that movement affects beat perception. Todd, Cousins, and Lee (2007) found that 16\% of variation in preferred beat rate can be predicted from anthropometric factors, such as weight as well as length and width of certain body segments. Phillips-Silver and Trainor found that both infants' (2005) and adults' (2007) encoding of meter can be affected by movement. Todd, O'Boyle, and Lee (1999) proposed that pulse is an inherently sensorimotor phenomenon in the sense that pulse perception necessarily involves motor system activity. Phillips-Silver and Trainor (2008) investigated whether passive movement of the head and legs affects the perception of rhythm, and found that head movement biases meter perception while movement of legs has no effect. Moreover, Trainor, Gao, Lei, Lehtovaara, and Harris (2009) discovered that galvanic stimulation of the vestibular system can be used to disambiguate an ambiguous metric pattern. These results suggest that the vestibular system has a central role in the perception of rhythm.

While there exists a few studies on synchronization with a musical beat (Drake et al., 2000; Large et al., 2002; Repp, 2005a, 2005b; Snyder \& Krumhansl, 2001; Toiviainen \& Snyder, 2003), the kinematic aspects of this activity have been investigated to a lesser extent. However, Eerola, Luck, and Toiviainen (2006) investigated toddlers' corporeal synchronization with music using a high resolution motion capture system. They found that 2-4 year old children exhibited periodic movement, and that this movement was at times synchronized with the tactus of music. The tactus refers to the basic pulse of music, i.e., the pulse to which listeners typically entrain when tapping. The periodic movement was most clearly visible in the vertical dimension. The embodiment of higher metrical levels in musical movement has received less attention. Naveda and Leman (2010), however, studied how the metric hierarchy of Samba and Charleston is reflected in professional dancers' repetitive gestures. To this end, they propose a method based on principal components analysis and periodicity transform (Sethares \& Staley, 2001). With respect to musical movement by ordinary music listeners, the presence of metrical levels has, to date, not been studied.

The present work investigates the nature of musicinduced movements, focusing on how pulsations on different metrical levels manifest in these movements. We were interested in two main questions. First, whether music-induced movements display movement components that are simultaneously synchronized to different metrical levels. Second, whether the synchronized movement patterns differ between the metrical levels. To this end, we applied kinetic analysis, body modeling, dimensionality reduction, and signal processing to data acquired using a high resolution motion capture system to identify the most typical movement patterns, or eigenmovements, synchronized to different metrical levels.

Based on the theoretical considerations presented above, in particular the notion of embodied cognition, we expect to see simultaneous synchronization of movement patterns to several metrical levels. Moreover, due to inertial and biomechanical properties of the body, we expect to see the higher (i.e., slower) metrical levels to be embodied as gross body movements, in particular movement of the torso, and lower (faster) metrical levels embodied as movements of the extremities. This behavior can be expected based on the simple argument that the specific period of oscillation of a rigid physical body is proportional to the square root of its moment of inertia and the moment of inertia is proportional to the mass and the physical dimensions of the rigid body. For instance, the torso has larger physical dimensions and larger mass than the arm and therefore has a higher moment of inertia and consequently a longer specific period of oscillation.

\section{Method \\ Participants}

A total of 18 participants ( 12 females, 6 males, mean age $=$ $24.2, S D$ of age $=3.9$ ) took part in the study. These participants were predominantly Finnish university students $(75 \%)$ with the remainder comprising international exchange students of various nationalities. The average number of years of formal music training was $8.0(S D=$ $6.7) ; 89 \%$ of those with music training had trained for 10 years or more. Most participants stated that they enjoyed dancing at home or in a nightclub (56\%), with an average 
time spent dancing of 2.0 hours per week $(S D=2.6$ hours), while a minority (25\%) of the participants had had formal dance or aerobics training. Additionally, participants were asked how many hours per week they spent doing physical activities or sports, of which the average time was 5.1 hours ( $S D=4.1$ hours). Participants received an honorarium in the form of a movie ticket for their participation in the study.

\section{Apparatus}

Participants' movements were recorded using an eightcamera optical motion capture system (Qualisys ProReflex). The system tracked the movement of reflective markers placed on each participant at a frame rate of $60 \mathrm{~Hz}$. The trajectories were interpolated together to create a three dimensional point-light display of each participant. The motion capture data were synchronized with the musical stimulus using the synchronization pulses transmitted by the Qualisys cameras. The musical stimulus was played back from a Pure Data (Pd) patch running on an Apple computer. Both the audio and the synchronization pulses were routed to the inputs of a Digidesign Mbox and recorded using ProTools software. For reference purposes, the sessions were additionally videotaped using a Panasonic Mini DV camcorder.

\section{Materials and Procedure}

Participants were recorded individually, and, prior to each motion capture session, a total of 28 reflective markers were attached to the participant's body. The locations of the markers were as follows $(\mathrm{L}=$ left, $\mathrm{R}=$ right, $\mathrm{F}=$ front, $\mathrm{B}=$ back): 1 : $\mathrm{LF}$ head (L frontal eminence); 2: RF head ( $\mathrm{R}$ frontal eminence); 3: LB head ( $\mathrm{L}$ dorsal parietal bone); 4: RB head (R dorsal parietal bone); 5: L shoulder (L scapular acromion); 6: R shoulder (R scapular acromion); 7: spine (midpoint between the superior angles of the scapulae); 8: breastbone (sternum); 9: LF hip (L anterior superior iliac spine); 10: RF hip ( $\mathrm{R}$ anterior superior iliac spine); 11: LB hip (L posterior superior iliac spine); 12: RB hip (R posterior superior iliac spine); 13: L elbow (L olecranon); 14: R elbow (R olecranon); 15: L inner wrist(L distal radius); 16: L outer wrist (L distal ulna); 17: $\mathrm{R}$ inner wrist ( $\mathrm{R}$ distal radius); 18: $\mathrm{R}$ outer wrist ( $\mathrm{R}$ distal ulna); 19: $\mathrm{L}$ middle finger (metacarpophalangeal joint of $\mathrm{L}$ digitus medius); 20: $\mathrm{R}$ middle finger (metacarpophalangeal joint of $\mathrm{R}$ digitus medius); 21: L knee (L lateral distal femur); 22: $\mathrm{R}$ knee (R lateral distal femur); 23: L ankle (L lateral malleolus); 24: $\mathrm{R}$ ankle ( $\mathrm{R}$ lateral malleolus); 25: L little toe (L 5th proximal phalanx); 26: L big toe (distal
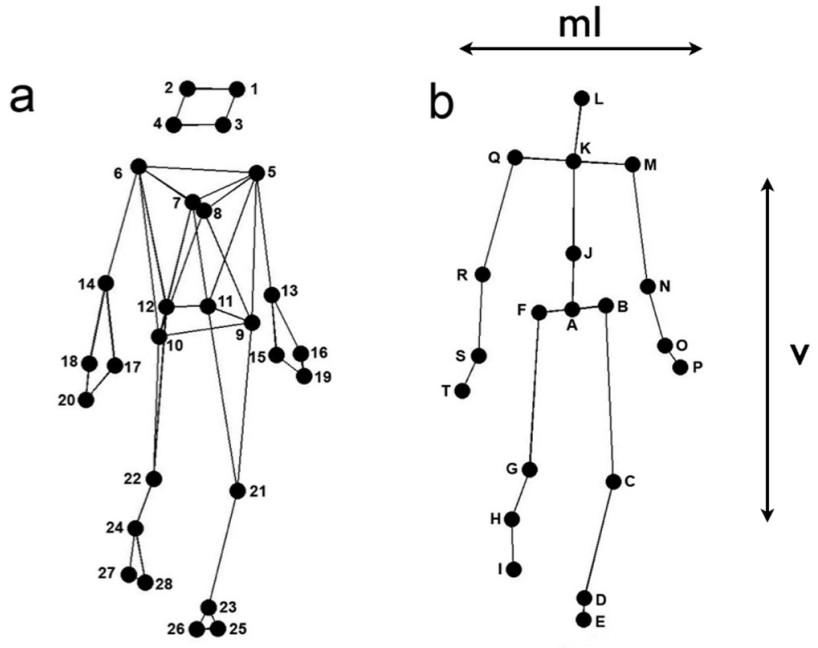

FIGURE 1. (a) Anterior view of the location of markers attached to participants' bodies. (b) Anterior view of the locations of the secondary markers used in the analysis (see text). Neighboring joints in kinematic chains are connected with lines. The directions of the mediolateral $(\mathrm{ml})$ and vertical ( $v$ ) axes are indicated by arrows. The anteroposterior axis is vertical to the surface of the page.

interphalangeal joint of $\mathrm{L}$ hallux); 27: $\mathrm{R}$ little toe ( $\mathrm{R}$ 5th proximal phalanx); 28: $\mathrm{R}$ big toe (distal interphalangeal joint of $\mathrm{R}$ hallux). The locations of the markers are depicted in Figure 1a.

Once the markers were attached and well placed, we ensured that they were nonintrusive to the participant's mobility, and that the participant was comfortable with the apparatus and the setting. The participant stood in the centre of the capture area and was instructed to 'move freely' to the music.

Participants were presented with an instrumental 12-bar blues progression performed in a minor key and $4 / 4$ meter. During the piece, the progression was repeated at four different tempi chosen randomly from the tempi $92,103,115,126$, and 138 beats per minute (bpm), and presented in random order. The total amount of motion capture data collected and subjected to analysis was ca. $37.5 \mathrm{~min}$.

\section{Results}

A schematic representation of the entire analysis procedure is provided in Figure 2.

In what follows, the steps of the analysis process are described in detail.

\section{Preprocessing}

To start the computational analysis, a set of secondary markers, subsequently referred to as the joints, was 


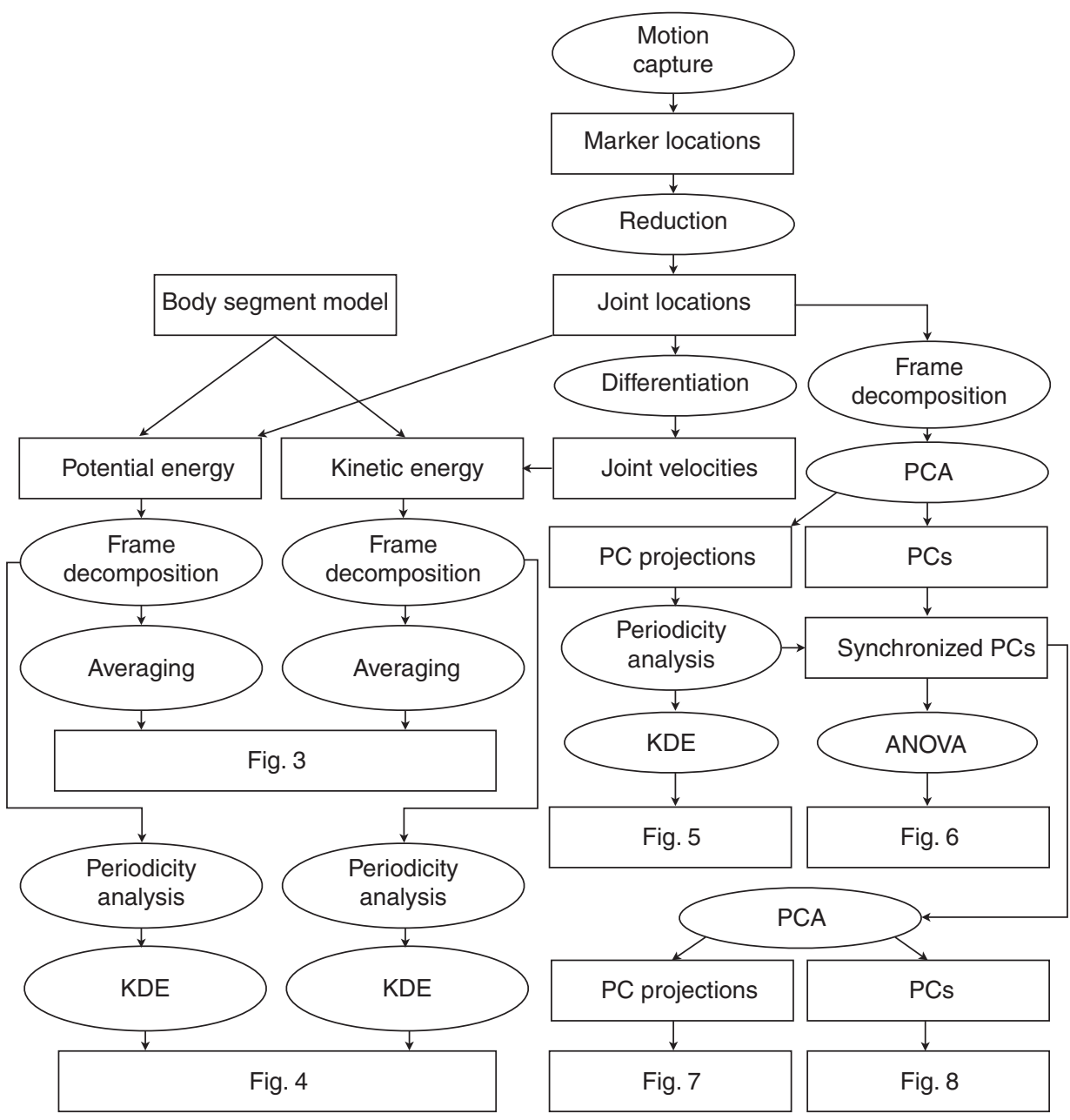

FIGURE 2. Overview of the analysis of the data. $\mathrm{PCA}=$ principal components analysis, $\mathrm{PC}=$ principal component, $\mathrm{KDE}=$ kernel density estimation.

derived from the marker locations. This was done in order to make the movement data compatible with the body-segment model used in subsequent analyses. The new set of twenty joints is depicted in Figure 1b. The locations of joints C, D, G, H, M, N, P, Q, R, S, and T are identical to the locations of one of the markers, while the locations of the other joints were obtained by averaging the locations of two or more markers. In addition to the joints obtained by the calculation described above, Figure $1 \mathrm{~b}$ displays as lines the body segments that connect the joints. The following nomenclature will be used in subsequent discussion of the joints: A: root; B: left hip; C: left knee; D: left ankle; E: left toes; F: right hip; G: right knee; H: right ankle; I: right toes; J: midtorso; K: manubrium; L: head; M: left shoulder; N: left elbow; O: left wrist; P: left fingers; Q: right shoulder; R: right elbow; S: right wrist; $T$ : right fingers.
The body segments considered in this study comprise five kinematic chains of body segments connected by joints. These are (see Figure 1b): ABCDE (left hip; left thigh; left leg; left foot), AFGHI (right hip; right thigh; right leg; right foot), AJKL (abdomen; thorax; head and neck), AJKMNOP (abdomen; thorax; left shoulder; left upper arm; left forearm; left hand), and AJKQRST (abdomen; thorax; right shoulder; right upper arm; right forearm; right hand).

\section{Mechanical Energy}

Next, we estimated two kinetic variables from the joint locations and velocities: potential energy and kinetic energy. Potential energy of a body of mass is a form of mechanical energy that depends on its height in the gravitational field. For instance, bending the knees while 
standing reduces the total potential energy of the body. Kinetic energy, on the other hand, is energy due to motion and thus depends on the speed of body of mass. The potential energy of the body was calculated using the formula

$$
E_{p o t}(t)=M \sum_{s} m_{s} g y_{s, c}(t)
$$

where $M$ stands for the total mass of the body, $m_{s}$ the mass of body segment $s$ relative to the total mass of the body, $g$ the acceleration of gravity, and $y_{s, c}$ the vertical location of the center of mass of body segment $s$. The kinetic energy was calculated using the formula

$$
\begin{aligned}
E_{\text {kin }}(t) & =E_{\text {trans }}(t)+E_{\text {rot }}(t) \\
& =M \sum_{s}\left(\frac{1}{2} m_{s} v_{s, c}^{2}(t)+\frac{1}{2} I_{s, c} \omega_{s}^{2}(t)\right),
\end{aligned}
$$

where $E_{\text {trans }}$ and $E_{\text {rot }}$ denote translational and rotational energy, respectively, $v_{s, c}$ the speed of the center of mass of body segment $s, I_{s, c}$ the moment of inertia of body segment $s$, and $\omega_{s}$ the angular velocity of body segment $s$.

To estimate the instantaneous velocities of the joints and the angular velocities of the body segments needed for the calculation of kinetic energy, we utilized numerical differentiation to the joint location data. To this end, we used the Savitzky-Golay smoothing FIR filter (Savitzky \& Golay, 1964) with a window length of seven samples and a polynomial order of two. These values were found to provide an optimal combination of precision and smoothness in the time derivatives.



The inertial constants of the body were obtained from the body segment model proposed by Dempster, Gabel, and Felts (1959; see also Robertson, Caldwell, Hamill, Kamen, \& Whittlesley, 2004). The model specifies a number of parameters for each of the body segments. These are the mass of the segment in relation to the total body mass, the distance of the center of mass from the proximal joint in relation to the segment length, and the radius of gyration in relation to segment length with respect to the center of mass, the proximal joint, and the distal joint.

To obtain an overview of the relationship of the total instantaneous kinetic and potential energies and the metrical structure of the musical stimulus, we decomposed the estimated energies into segments, each of which had a length equal to eight beats of the respective musical stimulus. Subsequently, the instantaneous energies were averaged across participants and across eightbeat segments in the stimuli. The results are shown in Figure 3. As can be seen, the average potential energy displays a clear periodicity at the one-beat level, the beat location closely matching the point of maximal decrease in potential energy and thus maximal downward velocity of the body. Average kinetic energy, on the other hand, displays a clear superposition of half-beat and two-beat periods.

Subsequently, we performed a periodicity analysis of the two components of mechanical energy. To this end, we first decomposed each motion capture recording into eight-beat sections with $50 \%$ overlap between neighboring sections. Subsequently, we estimated the period of potential and kinetic energy in each eight-beat segment using autocorrelation, which is a standard method for

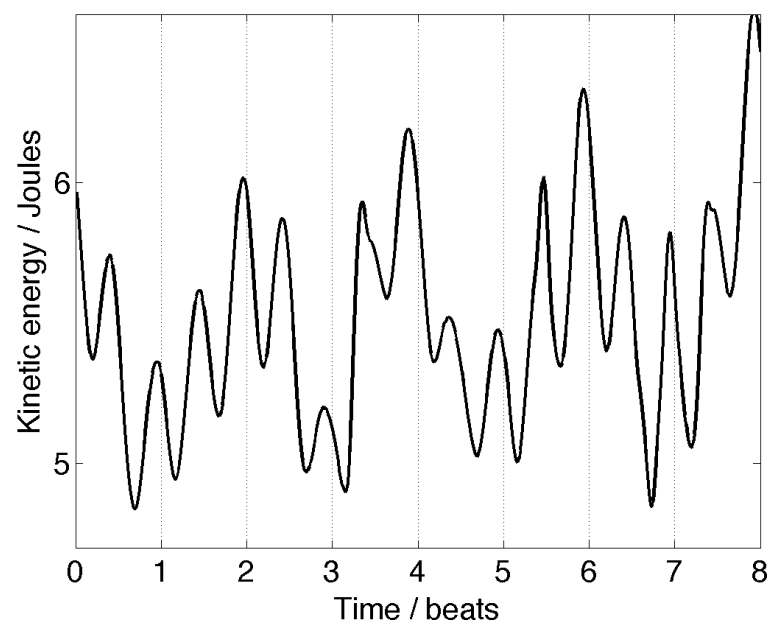

FIGURE 3. Total potential (left) and kinetic (right) energy of the body, averaged across participants and eight-beat segments. 

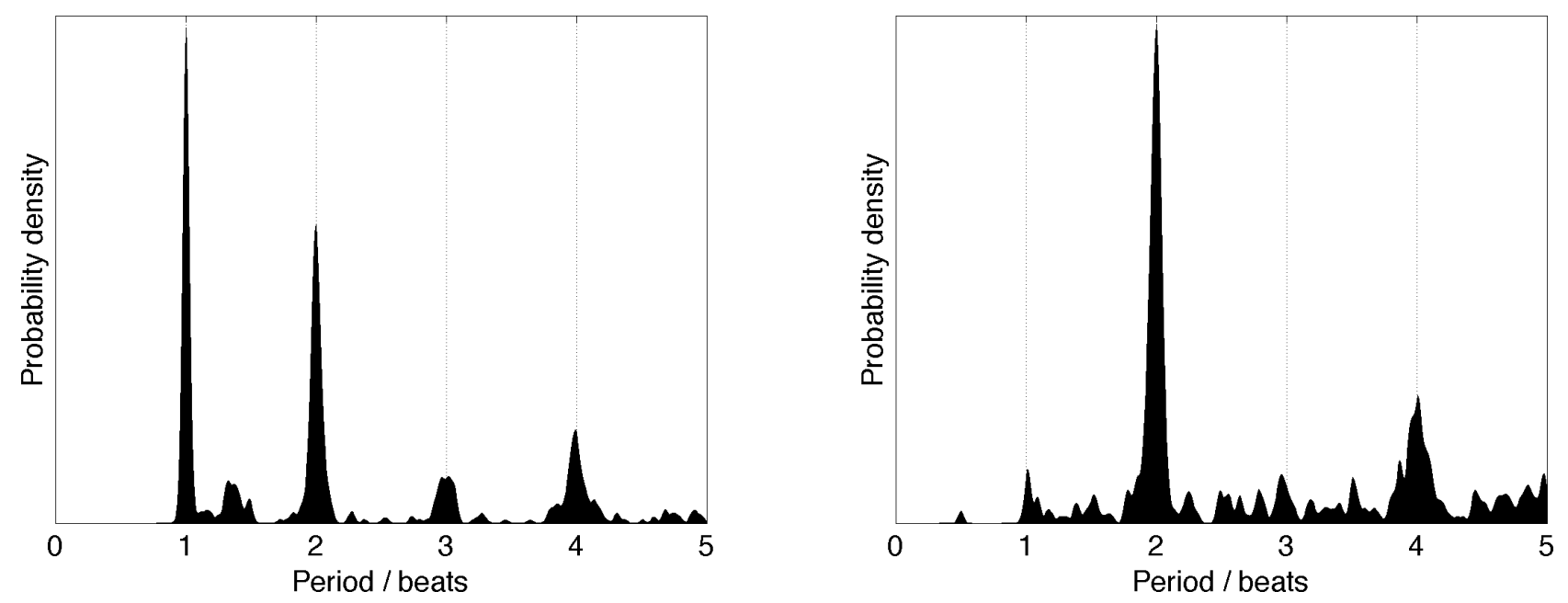

FIGURE 4. Kernel density estimate of distribution of periodicities for (a) potential energy and (b) kinetic energy.

periodicity estimation (Rabiner \& Schafer, 1978). In the autocorrelation analysis, the period was defined to be equal to the lag corresponding to the first maximum in the autocorrelation function. Subsequently, we estimated the distribution of the thus obtained periods using Kernel Density Estimation (KDE; Silverman, 1986). KDE is a nonparametric method for estimating the probability distribution of a variable from a sample, and is based on summation of kernel function (such as narrow Gaussian curves) centered at each value in the sample. The thus estimated distributions of the periods of potential and kinetic energy are displayed in Figure 4.

As is evident from Figure 4, both forms of mechanical energy display periodicities at the three main metrical levels corresponding to periods of one, two, and four beats. For potential energy (Figure 4a), the most common periodicity for each tempo corresponds to the length of one beat, with a two-beat period being the second most prominent. For kinetic energy (Figure $4 \mathrm{~b}$ ), the most common period is two beats, while four-beat periods are also relatively common.

It is noteworthy that the potential energy (Figure 4a) shows a relatively prominent peak at the period of two beats, while this period is not clearly visible in Figure $3 \mathrm{a}$. Similarly, for kinetic energy, the peak in Figure $4 \mathrm{~b}$ at the period of half a beat is relatively small, while this periodicity is clearly visible in Figure $3 \mathrm{~b}$. These differences are due to the different methods used to produce these graphs. In Figure 3 the graphs have been obtained by averaging the mechanical energies across the eight-beat segments, while those in Figure 4 have been obtained by picking the most prominent periodicity in each eightbeat segment and estimating the distribution of these values. Thus, it may happen, for instance, that although the half-beat period is often present in the kinetic energy, it is mostly less strong than the two-beat period and is thus seldom picked as the strongest periodicity.

The graphs of Figure 4 suggest that for most of the eight-beat segments, the strongest periodicity lies in the vicinity of the period of one metrical level (one, two, or four beats). Therefore, in terms of mechanical energy, the movements were synchronized to some metrical level for most of the time.

\section{Eigenmovements}

As the next step in the analysis, we extracted typical movement patterns from the data and investigated their periodicities. To this end, we used Principal components analysis (PCA). PCA is a method that transforms a large group of variables into a reduced group of uncorrelated variables called principal components (PCs), which are linear combinations of the original variables. The first PC accounts for as much of the variance in the data as possible and the successive PCs each in turn account for as much of the remaining variance as possible. As a result of PCA, each observation in the data set can be expressed as values of the reduced set of variables, called the PC projections.

Again, the data were decomposed into eight-beat sections with $50 \%$ overlap between neighboring sections. This decomposition was carried out because many of the participants changed their movement patterns during the presentation of the stimuli; applying PCA to the whole duration of a stimulus would not have produced meaningful results. The decomposition resulted in 44 
sections for each participant (11 sections per each tempo) and thus a total of 792 segments. For each segment, the motion capture data were rotated around the vertical axis so that the orientation of the frontal plane of the body, defined by the hip markers, was parallel to the first axis of the coordinate system. Subsequently, to reduce the dimensionality of the data, a PCA was carried out separately for each participant and each section. The data subjected to PCA thus consisted of a series of 60-component vectors. Each vector consisted of the three Euclidean coordinates of each of the 20 joints and there was one vector for each motion capture frame. The PCs obtained from this analysis were thus 60-component vectors, whose components indicated the amount to which each joint moved in each of the three directions in the respective movement pattern. The PC projections, on the other hand, were time series indicating how the movement pattern represented by the respective PC evolves in time.

\section{Periodicity of Eigenmovements}

For each segment, the first five PCs were included in the analysis. They accounted on average for $96.7 \%$ of the variance in each segment $(S D=2.3 \%)$. The PC projections from each PCA were subjected to a periodicity analysis using autocorrelation. Again, we estimated the distribution of the periods of the Principal Component projections using kernel density estimation. The result is shown in Figure 5. As can be seen, the most common periods correspond to the three main metrical levels (i.e., periods of one, two, and four tactus beats), with the twobeat period being the most prevalent, followed by four beats and one beat, in this order.

Next, we investigated the prevalence of eigenmovements embodying each metrical level. To this end, we compared the estimated periods of the eigenmovements with the period of one, two, and four beats of the respective musical stimulus. We found that $12 \%$ of the segments contained a PC that was synchronized to the one-beat level, in the sense that its period differed from that of one musical beat by less than $10 \%$. For the two-beat and four-beat levels, these proportions were $47 \%$ and $34 \%$, respectively. On average, the synchronized eigenmovements comprised



FIGURE 5. Distribution of periods of eigenmovements. a total of $29 \%$ of the total kinetic energy of the movements. For $65 \%$ of the segments, only one of the eigenmovements coincided with the period of any of the three metrical levels. According to this criterion, the participants thus embodied only one metrical level at a time for most of the segments. The eigenmovements synchronized to the three main metrical levels will be subsequently referred to as eigenmodes.

\section{Structure of Eigenmodes}

As a next step in the analysis, we investigated whether the eigenmodes embodying each of the three metrical levels differed from each other. More specifically, the aim of this analysis was to investigate whether there were differences between the metrical levels in terms of the movement of each joint along each of the three directions (mediolateral, anteroposterior, vertical).

To investigate these differences, we first performed a one-way ANOVA on each of the 60 components of the PCs. For the purpose of the analysis, for each participant and each metrical level, the PCs whose projections displayed a periodicity that was within $\pm 10 \%$ of the length of the respective period of the metrical level where chosen. Subsequently, we calculated for each of the 60 components the average value across the selected PCs. By doing this we obtained one averaged PC for each of the 18 participants and for each of the three metric levels.

For the calculation of the PC averages, absolute values of the components were used. This was because the PCs are only defined up to a multiplicative constant (Strang, 1976). For instance, if $\mathbf{p}$ is a PC obtained from a data set, so is -p. Therefore, if absolute values were not used, the averaging may have cancelled out some PCs having opposite signs. The ANOVAs thus regarded the amount of movement of each joint along each of the three directions without any regard to the direction of movement.

Due to the large number of comparisons (60), we used Bonferroni correction. The level of significance was thus set to $.05 / 60=.00083$. Table 1 displays the joints and movementdirections that showed a significant effect of metrical level in the amount of movement.

The results of the posthoc tests for each comparison and each direction are displayed graphically in Figure 6. In this figure, the joints displaying significant differences are shown as black circles. To summarize the findings displayed in this figure, the main significant difference between the one-beat and two-beat eigenmodes (panels $a$ and $b$ ) is that one-beat eigenmodes displayed more movement of shoulders in the vertical direction. Posthoc tests between one-beat and four-beat eigenmodes (panels $\mathrm{c}$ and $\mathrm{d}$ ) reveal that one-beat eigenmodes are 
TABLE 1. Results from ANOVA Testing Differences in the Amount of Movement Between the Three Metrical Levels. Only Significant Effects Are Shown.

\begin{tabular}{llcrc}
\hline \multirow{2}{*}{ Direction } & Joint & $\begin{array}{c}\text { Joint } \\
\text { symbol }\end{array}$ & $F(2,51)$ & $p$ \\
\hline Vertical & Root & $\mathrm{A}$ & 10.07 & .00026 \\
& Left hip & $\mathrm{B}$ & 10.05 & .00027 \\
& Right hip & $\mathrm{F}$ & 12.57 & .00005 \\
& Manubrium & $\mathrm{K}$ & 10.59 & .00019 \\
& Head & $\mathrm{L}$ & 13.57 & .00003 \\
& Right shoulder & $\mathrm{Q}$ & 11.57 & .00010 \\
& Right elbow & $\mathrm{R}$ & 9.17 & .00050 \\
& Right wrist & $\mathrm{S}$ & 9.32 & .00045 \\
& Left shoulder & $\mathrm{M}$ & 12.93 & .00004 \\
& Left elbow & $\mathrm{N}$ & 9.69 & .00035 \\
& Left knee & $\mathrm{C}$ & 8.82 & .00063 \\
& Root & $\mathrm{A}$ & 10.84 & .00016 \\
& Manubrium & $\mathrm{K}$ & 16.08 & .00001 \\
& Head & $\mathrm{L}$ & 10.97 & .00015 \\
& Right shoulder & $\mathrm{Q}$ & 10.21 & .00024 \\
& Right elbow & $\mathrm{R}$ & 9.63 & .00036 \\
& Left shoulder & $\mathrm{M}$ & 20.42 & .00000 \\
& Left hip & $\mathrm{B}$ & 12.08 & .00007 \\
& Right elbow & $\mathrm{R}$ & 9.86 & .00031 \\
& Right wrist & $\mathrm{S}$ & 9.28 & .00046 \\
& Left wrist & $\mathrm{O}$ & 11.76 & .00009 \\
& & & &
\end{tabular}

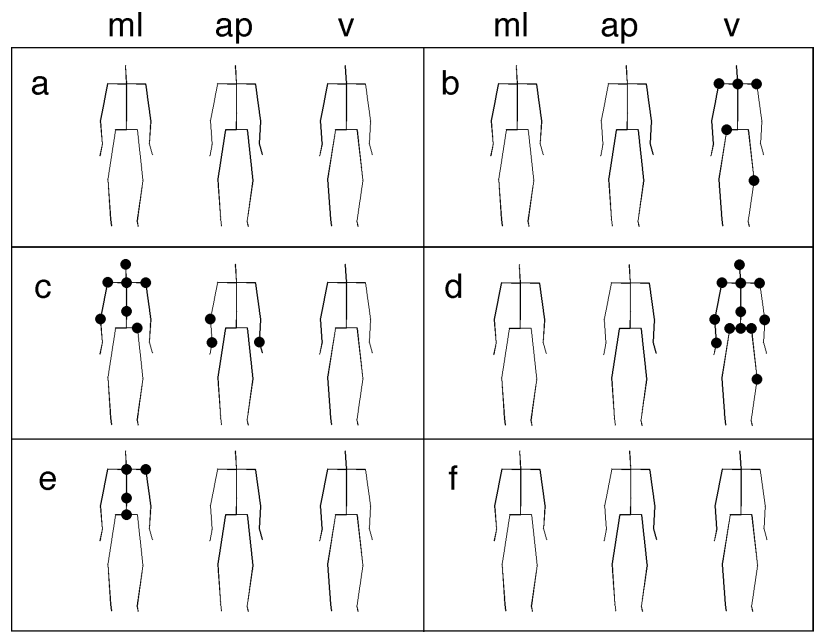

FIGURE 6. Significant differences in the amount of movement of each joint along each direction between eigenmodes $(\mathrm{ml}=$ mediolateral direction; $\mathrm{ap}=$ anteroposterior direction; $\mathrm{v}=$ vertical direction). For each comparison and each direction, joints having significant differences are displayed with black circles; (a) less movement in one-beat eigenmodes than in two-beat eigenmodes; (b) more movement in one-beat eigenmodes than in two-beat eigenmodes; (c) less movement in one-beat eigenmodes than in four-beat eigenmodes; (d) more movement in onebeat eigenmodes than in four-beat eigenmodes; (e) less movement in two-beat eigenmodes than in four-beat eigenmodes; $(f)$ more movement in two-beat eigenmodes than in four-beat eigenmodes. associated with less movement of the upper torso in the mediolateral direction and hands in the anteroposterior direction than four-beat eigenmodes, while one-beat eigenmodes are associated with more movement of torso and arms in the vertical direction than four-beat eigenmodes. Finally, two-beat eigenmodes showed less movement of the torso in the mediolateral direction than four-beat eigenmodes (panels e and $\mathrm{f}$ ).

\section{Between-Subjects PCA}

To investigate more closely the nature of different movement patterns at the different metrical levels, a second (between-subjects) PCA was carried out on the one-beat, two-beat, and four-beat eigenmodes. In other words, the PCs obtained from the PCA described above, whose projections where synchronized to one of the three metrical levels (allowing a tolerance of $10 \%$ of the length of musical beat), were subjected to the betweensubjects PCA.

For subsequent analysis we decided to include PCs that contained a minimum of $75 \%$ of the variance. Using this criterion, we ended up with six PCs that contained $76.5 \%$ of the variance. The PCs obtained from this second-order PCA subsequently are referred to as the second-order PCs. To assess the degree to which each of the second-order PCs represents movement patterns synchronized to each of the metrical levels, the proportion of variance contained in the second-order PC component scores within each of the three metrical levels was quantified. The rationale behind this procedure was that if the second-order component scores for a given metric level display a high amount of variance, the respective second-order PC has a high degree of similarity to the first-order PCs representing this metric level. This follows from the fact that the PC component score is obtained by calculating the dot product between the first-order PC and the second-order PC, and since the PCs are normalized to have unit length, the dot product is equal to the cosine of the angle between first-order PCs and the second-order PC. Thus, such a second-order PC can be regarded to represent a typical movement pattern for the metric level.

The proportion of variance contained in the projections onto the first PCs are shown in Figure 7. As is evident from this figure, the metrical levels differ in terms of the variance contained in each of the PC projections. In particular, the eigenmovements synchronized to the one-beat level retain the highest proportion of variance when projected onto PCs 3 and 5. Similarly, the eigenmovements synchronized to the two-beat and four-beat levels retain the highest proportion of 

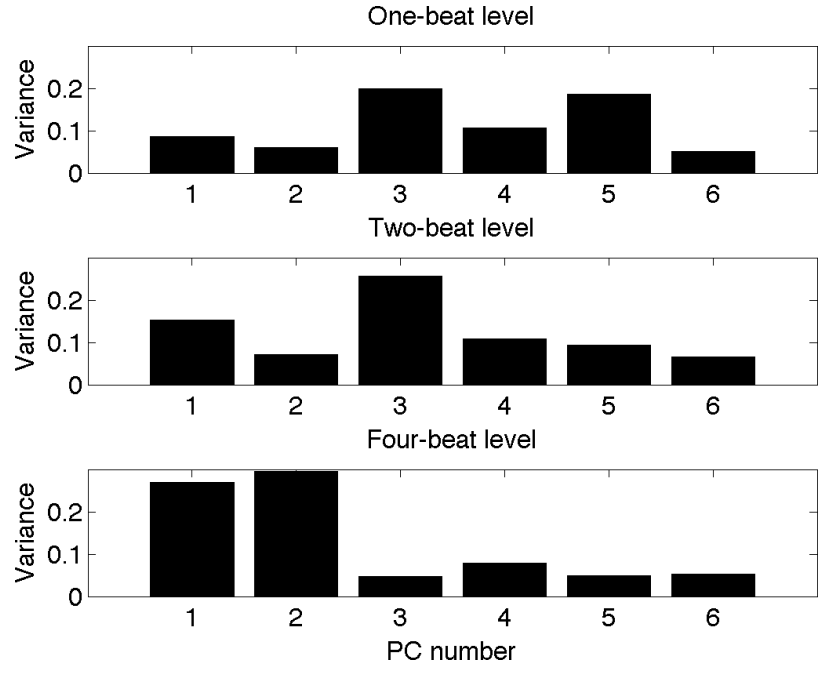

FIGURE 7. Proportion of variance contained in the projections of the primary PCs onto the secondary PCs for each metrical level.

variance when projected onto PCs 3 and 1, and 2 and 1 , respectively.

The six secondary PCs are graphically depicted in Figure 8. In what follows, PCs 1, 2, 3, and 5, which show the highest variances in Figure 7, are analyzed in detail. PC 1 contains high values in the anteroposterior components of the shoulders, elbows, wrists, and fingers (gray bars at joints M-T). Moreover, the components corresponding to the two sides of the body (M-P vs. $\mathrm{Q}-\mathrm{T}$ ) have opposite signs. PC 1 thus corresponds to the rotation of the upper torso. PC 2 displays large values in the mediolateral components of most joints (black bars). Moreover, the components have the same sign. Therefore, PC 2 corresponds to lateral swaying of the body. PC 3 displays largest values in the mediolateral components of the elbows, wrists, and fingers (black bars at joints $\mathrm{N}-\mathrm{P}$ and R-T). Again, the components corresponding to the two sides of the body (N-P vs. R-T) have opposite signs. Therefore, PC 3 corresponds to antiphase mediolateral arm movement. Finally, PC 5 shows largest values in the vertical components of elbows, wrists, and fingers (joints N-P and R-T). Therefore, PC 5 corresponds to vertical movement of the arms.

Based on this analysis, we can identify the following typical movements for each of the metrical levels.

- One-beat level: (1) mediolateral arm movements (PC 3); (2) vertical arm movements (PC 5);

- Two-beat level: (1) mediolateral arm movements (PC 3); (2) rotation of the upper torso (PC 1);

- Four-beat level: (1) lateral swaying of the body (PC 2); (2) rotation of the upper torso (PC 1).

\section{Discussion}

We investigated music-induced movement, focusing particularly on the relationship between movement patterns and metrical levels of music. A kinetic analysis of peaks in mechanical energy (potential energy and kinetic energy) revealed that participants embodied the musical stimulus on several metrical levels. A subsequent kinematic analysis of the periodicity structure of the movement revealed that participants synchronized with periods of one, two, and four beats, supporting the findings from the kinetic domain. The analysis also showed that several metrical levels can be embodied simultaneously in the movement, although participants mostly tended to embody only one metrical level at a time. Regarding direction of movement at different metrical levels, we found that the tactus (one-beat level) tended to be embodied mostly as vertical movement, while the four-beat level tended to be embodied as mediolateral movement. This observation is consistent with the kinetic finding that the average potential energy of the body displays a period of one beat.

A more detailed kinematic analysis revealed that the tactus level often was associated with vertical hand and torso movements as well as mediolateral arm movements, the two-beat level with mediolateral arm movements and rotation of the upper torso, and the four-beat level with lateral flexion of the torso and rotation of the upper torso. This observation is in line with our hypothesis that faster metric levels are embodied in the extremities, and slower ones in the central parts of the body. Furthermore, this finding is in tune with the kinetic observation that the most prevalent period of kinetic energy is two beats. The reasons for this are as follows. First, the torso has a significant mass, and thus its movement contributes significantly to the body's total kinetic energy. Second, because in periodic motion kinetic energy obtains two maxima and two minima during one cycle, a two-beat period in kinetic energy corresponds to a four-beat period of movement.

An interesting observation was that, compared to twobeat and four-beat periods, one-beat period was relatively rare in the eigenmovements. There are two possible explanations for this finding. First, the method of Principal components analysis extracts movement patterns that display the highest variance. It is thus possible that there exist movement patterns with one-beat period, but they may possess a low degree of variance and are thus not discovered by this method. This finding also can be explained by the type of variable that was used in the analysis. In particular, we used marker locations in the analysis. Using some other kinematic variable, such as marker speed, would have resulted in different periodicity 


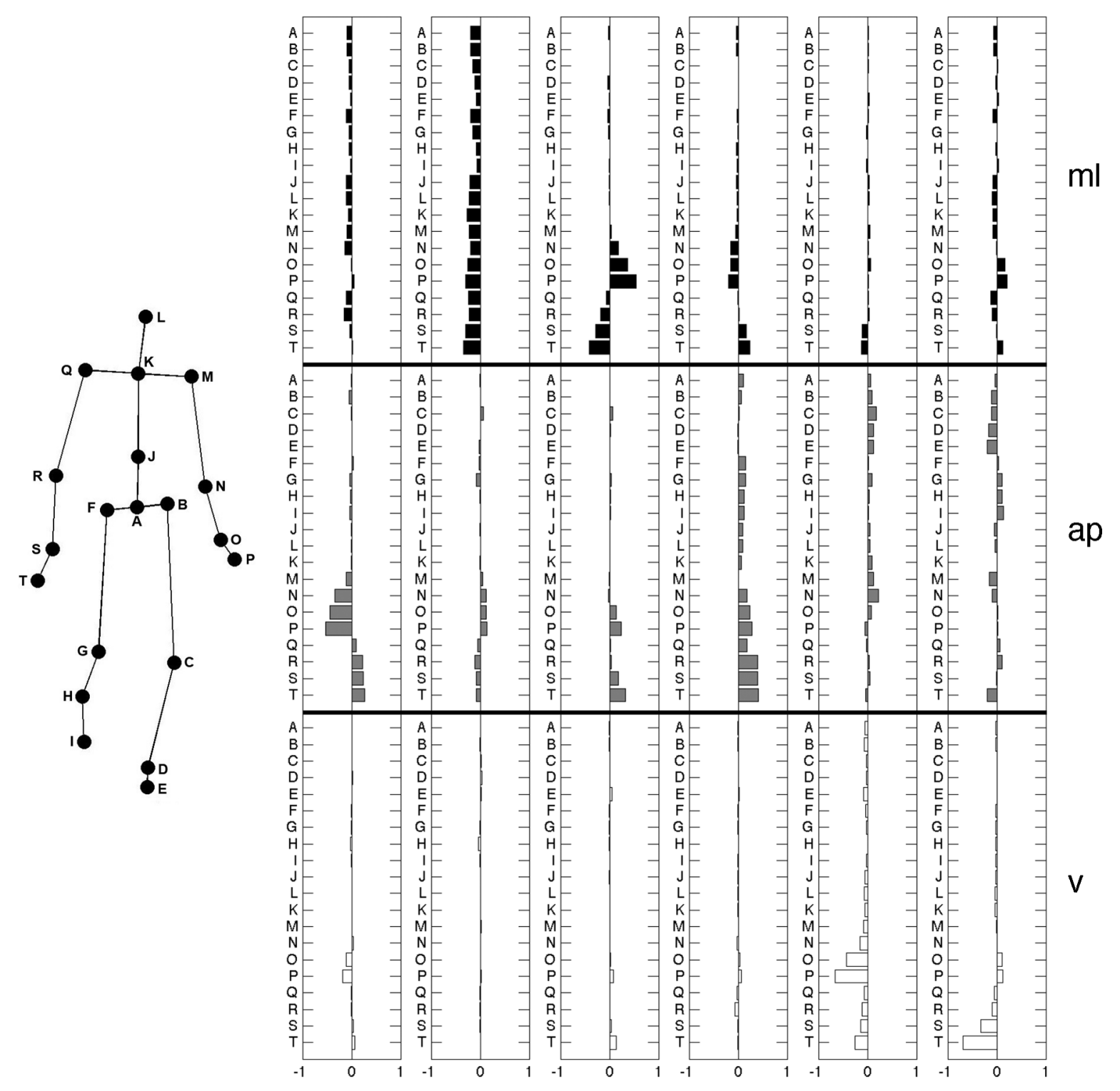

FIGURE 8. First six second-order PCs obtained from the analysis. The first PC is in the left and the sixth in the right. On the left side of each graph, the letters refer to the joints as depicted in the left of the figure. The components are shown separately for mediolateral (ml), anteroposterior (ap), and vertical (v) dimensions.

distributions. For instance, for a mediolateral swaying of the body with a two-beat period, the speed of the torso shows a period of one beat. Also, to produce such movement, overall muscular effort has a period of one beat (muscular effort is maximal at the points where the tilt of the body is maximal). Therefore, one can say that even if this kind of movement pattern shows a period of two beats in terms of marker locations, the one-beat level is embodied in the muscular activity.

A possible limitation of the present study is that it used only one musical stimulus, albeit presented with a range of different tempi. Consequently, it may be that the observed movement patterns represent some musical features characteristic to this stimulus and thus are not generalizable to other musical stimuli. In subsequent work, we plan to include stimuli representing other various musical genres and several time signatures.

One could assume that the tempo at which the stimulus was presented to the participant could have had an effect on the way they moved to the music. For instance, given our preference to move at frequencies close to $2 \mathrm{~Hz}$ (McDougall \& Moore, 2005), one could expect that 
stimuli whose tempi are in the vicinity of $120 \mathrm{bpm}$ would elicit larger movements than other stimuli. We compared the average kinetic energies of the participants across the different tempi, but failed to observe any significant differences. More research is needed to clarify this issue.

A further potential limitation of the present study is that the kinematic analysis focused entirely on the location of the markers, while ignoring other kinematic variables such as velocity, acceleration, and jerk. Analysis of these kinematic variables could potentially reveal patterns that remain hidden in the location-based analysis.

The use of principal components analysis for dimensionality reduction makes certain assumptions about the analyzed data that are not necessarily fulfilled by the present data. First, the Principal Components are noncorrelated, or orthogonal, and thus associating the eigenmodes with them makes the assumption that the movement patterns synchronized to the different metrical levels are orthogonal as well. This, however, may not be the case. Therefore, using nonorthogonal dimensionality reduction methods such as Independent Component Analysis (Hyvärinen, Karhunen, \& Oja, 2001) or Denoising Source Separation (Särelä \& Valpola, 2005) for eigenmode extraction could provide new insights into the problem.

A second assumption of principal components analysis is that the analyzed data are stationary within the analysis window. This may hold true when analyzing simple movement patterns such as walking, but with music-induced movement the patterns may change over time and the stationarity criterion is not met. This problem could be overcome by using eigenmode extraction methods that do not assume stationarity, such as Empirical Mode Decomposition (Huang et al., 1998).

The aforementioned limitations notwithstanding, our study is the first to tackle the question of music listeners' movement and embodiments of musical meter using quantitative methods. We have shown that several levels of metrical hierarchy simultaneously can be embodied in the movements. Furthermore, we have identified the most typical movement types of different metric levels. In the future, we expect to gain a deeper insight into this phenomenon using more varied stimulus sets and improved methods of analysis, as described above.

\section{Author Note}

We wish to thank Marc Leman and two anonymous reviewers for helpful comments, and Aki Tulikari for his help in collecting the motion capture data. This study was supported by the Academy of Finland (projects 118616, 125710, and 119959).

Correspondence concerning this article should be addressed to Petri Toiviainen, Department of Music, PO Box 35(M), 40014 University of Jyväskylä, Finland. E-MAIL: petri.toiviainen@jyu.fi

\section{References}

Arom, S. (1991). African polyphony and polyrhythm. Cambridge, UK: Cambridge University Press.

Cross, I. (2003). Music, cognition, culture and evolution. In I. Peretz \& R. J. Zatorre (Eds.), The cognitive neuroscience of music (pp. 42-56). New York: Oxford University Press.

Dempster, W. T., Gabel, W. C., \& Felts, W. J. L. (1959). The anthropometry of manual work space for the seated subject. American Journal of Physical Anthropology, 17, 289-317.

Drake, C., Penel, A., \& Bigand, E. (2000). Tapping in time with mechanically and expressively performed music. Music Perception, 18, 1-23.

Eerola, T., Luck, G., \& Toiviainen, P. (2006, August). An investigation of pre-schoolers' corporeal synchronization with music. Paper presented at the 9th International Conference on Music Perception and Cognition, Bologna, Italy.

Fraisse, P. (1982). Rhythm and tempo. In D. Deutsch (Ed.), The psychology of music (pp. 148-180). New York: Academic Press.

Huang, N. E., Shen, Z., Long, S. R., Wu, M. C., Shih, H. H., Zheng, Q., et AL. (1998). The empirical mode decomposition and the Hilbert spectrum for nonlinear and nonstationary time series analysis. Proceedings of the Royal Society of London. Series A, 454, 903-995.

Hyvärinen, A., Karhunen, J., \& OJA, E. (2001). Independent component analysis. New York: Wiley.

Large, E. W., Fink, P., \& Kelso, J. A. (2002). Tracking simple and complex sequences. Psychological Research, 66, 3-17.

Leman, M. (2008). Embodied music cognition and music mediation technology. Cambridge, MA: MIT Press.

LERDAHL, F., \& JACKENDOFF, R. (1983). A generative theory of tonal music. Cambridge, MA: MIT Press.

Lesaffre, M., De Voogdt, L., Leman, M., De Baets, B., De Meyer, H., \& Martens, J.-P. (2008). How potential users of music search and retrieval systems describe the semantic quality of music. Journal of The American Society for Information Science and Technology, 59, 695-707.

Liberman, A. M., \& Mattingly, I. G. (1985). The motor theory of speech-perception revised. Cognition, 21, 1-36.

McDougall, H. G., \& Moore, S. (2005). Marching to the beat of the same drummer: The spontaneous tempo of human locomotion. Journal of Applied Physiology, 99, $1164-1173$. 
Naveda, L., \& Leman, M. (2010). The spatiotemporal representation of dance and music gestures using topological gesture analysis (TGA). Music Perception, 28, 93-111.

Palmer, C., \& Krumhansl, C. L. (1990). Mental representations of musical meter. Journal of Experimental Psychology: Human Perception and Performance, 16, 728-741.

PARnCUTT, R. (1994). A perceptual model of pulse salience and metrical accent in musical rhythms. Music Perception, 11, 409-464.

Patel, A. D., Iversen, J. R., Chen, Y., \& Repp, B. H. (2005). The influence of metricality and modality on synchronization with a beat. Experimental Brain Research, 163, 226-238.

Phillips-Silver, J., \& Trainor, L. J. (2005). Feeling the beat: Movement influences infant rhythm perception. Science, 308, 1430.

Phillips-Silver, J., \& Trainor, L. J. (2007). Hearing what the body feels: Auditory encoding of rhythmic movement. Cognition, 105, 533-546.

Phillips-Silver, J., \& Trainor, L. J. (2008). Vestibular influence on auditory metrical interpretation. Brain and Cognition, 67, 94-102.

RABINer, L. R., \& SCHAFer, R. W. (1978). Digital processing of speech signals. Englewood Cliffs, NJ: Prentice-Hall.

Repp, B. H. (2005a). Rate limits of on-beat and off-beat tapping with simple auditory rhythms: 1 . Qualitative observations. Music Perception, 22, 479-496.

Repp, B. H. (2005b). Rate limits of on-beat and off-beat tapping with simple auditory rhythms: 2 . The roles of different kinds of accent. Music Perception, 23, 165-187.

Repp, B. H., \& Penel, A. (2004). Rhythmic movement is attracted more strongly to auditory than to visual rhythms. Psychological Research-Psychologische Forschung, 68, 252-270.

Robertson, D. G. E., Caldwell, G. E., Hamill, J., Kamen, G., \& WhitTlesley, S. N. (2004). Research methods in biomechanics. Champaign, IL: Human Kinetics.
SÄrelä, J., \& VAlpola, H. (2005). Denoising source separation. Journal of Machine Learning Research, 6, 233-272.

SAVitzky, A., \& Golay, M. J. E. (1964). Smoothing and differentiation of data by simplified least squares procedures. Analytical Chemistry, 36, 1627-1639.

Sethares, W. A., \& Staley, T. W. (2001). Meter and periodicity in musical performance. Journal of New Music Research, 30, 149-158.

Silverman, B. W. (1986). Density estimation for statistics and data analysis. London: Chapman Hall.

Snyder, J., \& Krumhansl, C. L. (2001). Tapping to ragtime: Cues to pulse finding. Music Perception, 18, 455-489.

Strang, G. (1976). Linear algebra and its applications. New York: Academic Press.

Styns, F., van Noorden, L., Moelants, D., \& Leman, M. (2007). Walking on music. Human Movement Science, 26, 769-785.

Todd, N. P. M., Cousins, R., \& Lee, C. S. (2007). The contribution of anthropometric factors to individual differences in the perception of rhythm. Empirical Musicology Review, 2, 1-13.

Todd, N. P. M., O’Boyle, D. J., \& Lee, C. S. (1999). A sensory-motor theory of rhythm, time perception and beat induction. Journal of New Music Research, 28, 5-28.

Toiviainen, P., \& Snyder, J. S. (2003). Tapping to Bach: Resonance-based modeling of pulse. Music Perception, 21, 43-80.

Trainor, L. J., Gao, X., Lei, J.-J., Lehtovaara, K., \& HARRIS, L. R. (2009). The primal role of the vestibular system in determining musical rhythm. Cortex, 45, 35-43.

Wallin, N. L., Merker, B., \& Brown, S. (2000). The origins of music. Cambridge, MA: MIT Press.

VAn Noorden, L., \& Moelants, D. (1999). Resonance in the perception of musical pulse. Journal of New Music Research, $28,43-66$. 